\title{
Clinical and genetic investigation of families with type II Waardenburg syndrome
}

\author{
YONG CHEN $^{1 *}$, FUWEI YANG ${ }^{2,3^{*}}$, HEXIN ZHENG $^{1}$, JIANDA ZHOU $^{4}$, \\ GANGHUA ZHU ${ }^{3}$, PENG $\mathrm{HU}^{3}$ and WEIJING WU ${ }^{3}$ \\ ${ }^{1}$ Key Laboratory of Genetics and Birth Health of Hunan, The Family Planning Institute of Hunan, Changsha, \\ Hunan 410126; ${ }^{2}$ Department of Otolaryngology, Meizhou People's Hospital, Meizhou, Guangdong 514031; \\ ${ }^{3}$ Department of Otolaryngology, The Second Xiangya Hospital of Central South University, Changsha, \\ Hunan 410011; ${ }^{4}$ Department of Burns and Plastic Surgery, Third Xiangya Hospital, \\ Central South University, Changsha, Hunan 410013, P.R. China
}

Received February 15, 2015; Accepted December 11, 2015

DOI: $10.3892 / \mathrm{mmr} .2016 .4774$

\begin{abstract}
The present study aimed to investigate the molecular pathology of Waardenburg syndrome type II in three families, in order to provide genetic diagnosis and hereditary counseling for family members. Relevant clinical examinations were conducted on the probands of the three pedigrees. Peripheral blood samples of the probands and related family members were collected and genomic DNA was extracted. The coding sequences of paired box 3 (PAX3), microphthalmia-associated transcription factor (MITF), sex-determining region Y-box 10 (SOX10) and snail family zinc finger 2 (SNAI2) were analyzed by polymerase chain reaction and DNA sequencing. The heterozygous mutation, c.649_651delAGA in exon 7 of the MITF gene was detected in the proband and all patients of pedigree 1; however, no pathological mutation of the relevant genes (MITF, SNAI2, SOX10 or PAX3) was detected in pedigrees 2 and 3 . The heterozygous mutation c.649_651delAGA in exon 7 of the MITF gene is therefore considered the disease-causing mutation in pedigree 1 . However, there are novel disease-causing genes in Waardenburg syndrome type II, which require further research.
\end{abstract}

\section{Introduction}

There are $>400$ types of syndromic hearing loss, and Waardenburg syndrome (WS) is the most common,

Correspondence to: Dr Yong Chen, Key Laboratory of Genetics and Birth Health of Hunan, The Family Planning Institute of Hunan, 1292 Yuanda Road, Changsha, Hunan 410126, P.R. China

E-mail: chenyong0008@sina.com

*Contributed equally

Key words: Waardenburg syndrome, clinical classification, genetic mutation accounting for $2-5 \%$ of congenital deafness (1). WS is a type of auditory-pigmentary syndrome, the clinical manifestations of which include congenital neurosensory deafness, change in iris pigmentation, telecanthus, abnormal distribution of hair and skin pigmentation (prematurely white hair, eyebrows and eyelashes at <30-years old; facial freckles; skin depigmentation leukoplakia), wide root of the nose, and synophrys. WS is classified into four types, according to various clinical manifestations.

The pathogenesis of WS is largely attributed to the mutations in genes including PAX3, MITF, SNAI2, SOX10, ENDRB, and EDN3. Dysregulation of these genes causes abnormal development of neural crest cells, change in iris pigmentation, deafness, hair graying, and abnormal skin pigmentation. Notably, the distinct subtypes of WS differ in the pathogenic mutations of relevant genes (2).

The present study investigated three WS pedigrees, and clinical classification was made according to the patients' symptoms. Written informed consent was obtained from the family members who were willing to participate in the present study. The known candidate disease-causing genes were selected for mutation detection, in order to obtain a genetic diagnosis. The current study was approved by the ethics committee of the Family Planning Institute of Hunan Province (Changsha, China)

\section{Materials and methods}

Pedigree 1. The proband was a patient at The Special Clinic of Neonate Hearing Screening, The Second Xiangya Hospital (Changsha, China), who planned to undergo fitting of a cochlear implant. The family consisted of three generations (nine individuals) with five cases of WS, including the proband, the proband's grandmother, mother, aunt and uncle (Fig. 1).

Pedigree 2. The proband was a patient at The Special Clinic of Neonate Hearing Screening, The Second Xiangya Hospital. The family consisted of three generations (14 individuals) with two cases of WS, including the proband and the proband's father (Fig. 2). 


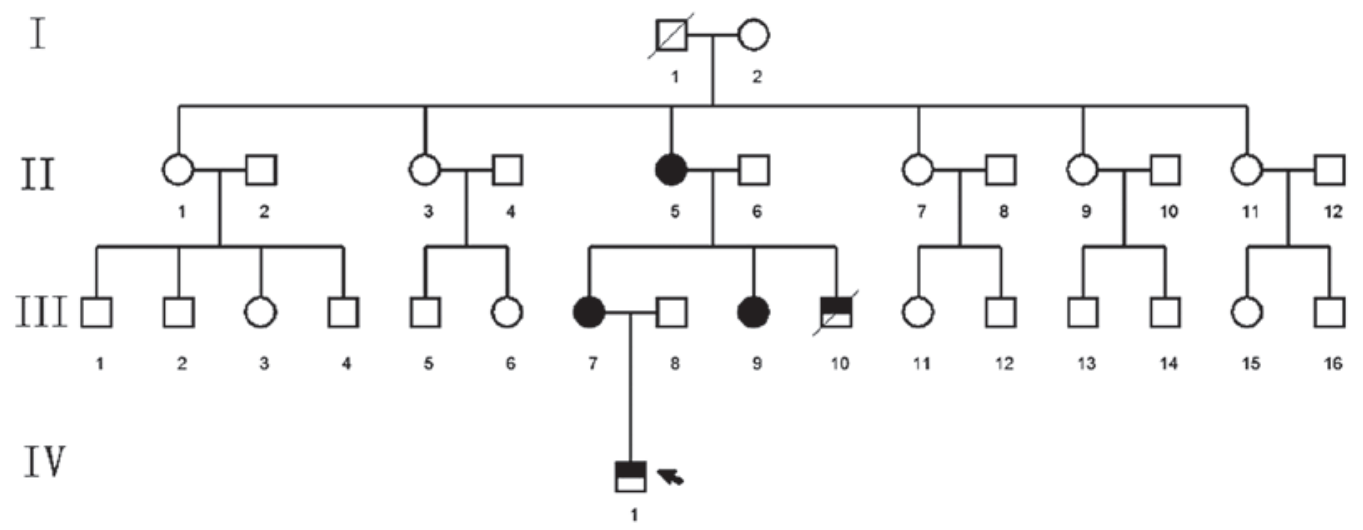

Figure 1. Chinese WS pedigree WS01. High clinical variability was observed within the family, and not all affected persons exhibited all clinical features. Circle, female; square, male. Filled quadrants indicate phenotype associated with WS: Lower left, premature graying hair; upper left, hearing loss; upper right, heterochromia iridis; lower right, pigmentation and freckles on the skin. A slash through the symbol indicates deceased individual. Arrow indicates the proband. WS, Waardenburg syndrome.

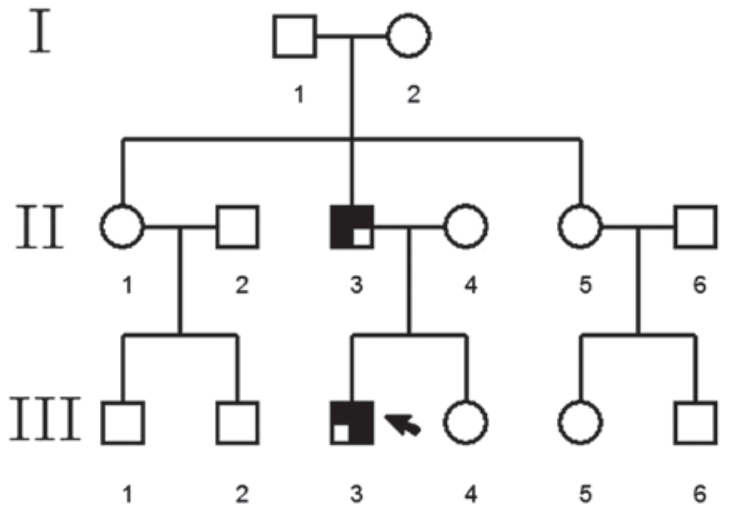

Figure 2. Chinese WS pedigree WS02. Clinical variability was observed within the same family. Not all affected persons exhibited all clinical features. Circle, female; square, male. Filled quadrants indicate phenotype associated with WS: Lower left, joint deformities; upper left, heterochromia iridis; upper right, graying hair on the forehead; lower right, hearing loss. Arrow indicates the proband. WS, Waardenburg syndrome.

Pedigree 3. The proband was a patient at The Special Clinic of Neonate Hearing Screening, The Second Xiangya Hospital, who planned to undergo fitting of a cochlear implant. The family consisted of three generations (four individuals) with two cases of WS, including the proband and the proband's father (Fig. 3).

Pedigree investigation and sample collection. According to the principle of informed consent, following approval from all family members, detailed examinations were performed on all patients by medical specialists. The following tests were conducted: Observation of skin pigmentation, hair color, joints, skeletomuscular system, digestion, nerves, ophthalmology and otology, and an assessment of intelligence. In addition, a detailed audiological examination was conducted on the probands. The clinical audiology assessment included pure tone test, acoustic immitance, auditory steady-state response, auditory brainstem response (ABR), otoacoustic emission, test of study ability and psychiatric behavior development, and ossa temporale computerized tomography and magnetic resonance imaging. Peripheral vein blood samples $(5 \mathrm{ml})$ were

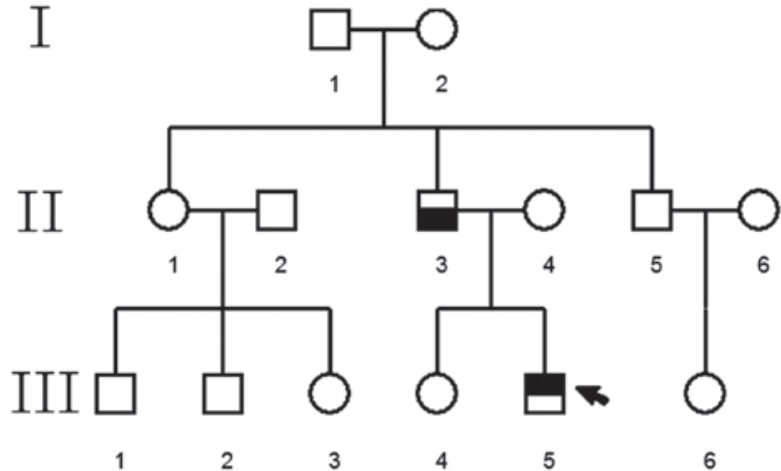

Figure 3. Chinese WS pedigree WS03. Clinical variability was observed within the same family. Not all affected persons exhibited all clinical features. Circle, female; square, male. Filled quadrants indicate phenotype associated with WS: Lower left, premature graying hair; upper left, hearing loss; upper right, heterochromia iridis; lower right, joint deformities. Arrow indicates the proband. WS, Waardenburg syndrome.

collected from all subjects and were placed in vacuum heparin anticoagulant tubes.

DNA extraction. Whole genomic DNA was isolated from the blood samples using the phenol-chloroform extraction, and was quantified by an ultraviolet spectrophotometer Du800 (Beckman Coulter, Inc., Brea, CA, USA). The DNA was subsequently stored at $-20^{\circ} \mathrm{C}$ until further use.

Analysis of genetic mutations. The probands and their healthy brother, sister or relatives were selected as the subject of mutation analysis. The sequences of the candidate genes, paired box 3 (PAX3), microphthalmia-associated transcription factor (MITF), sex-determining region Y-box 10 (SOX10), snail family zinc finger 2 (SNAI2), were used as the template. The online primer design software Primer 3 (http://primer3.ut.ee/) was used to design the polymerase chain reaction (PCR) primers to amplify all exons, and the PCR primers (Invitrogen; Thermo Fisher Scientific, Inc., Beijing, China) to amplify boundary sequence of exon/intron of the candidate gene (Tables I-IV). The amplification reaction was set in a total volume of $20 \mu \mathrm{l}$ containing the PrimeSTAR Max DNA polymerase and PCR 
Table I. Polymerase chain reaction primer sequence of the exons of paired box 3 gene.

\begin{tabular}{|c|c|c|c|}
\hline Exon & $\begin{array}{l}\text { Sequence of upstream } \\
\text { primer }\left(5^{\prime}-3^{\prime}\right)\end{array}$ & $\begin{array}{l}\text { Sequence of downstream } \\
\text { primer }\left(5^{\prime}-3^{\prime}\right)\end{array}$ & $\begin{array}{l}\text { Fragment } \\
\text { size (bp) }\end{array}$ \\
\hline 1 & ACTCGGTGTCACCACAGGA & CCTGGAAGCACCAAAGGAG & 564 \\
\hline 2 & TACGTGCTGCTGTTCTTTGC & TTACGCACCTTCACAAACCTC & 443 \\
\hline 3 & TCTGGTCTGCCCCTTTCTAA & ATTGGGGTGATTACGTCTGG & 389 \\
\hline 4 & GTCCAGAGATGCAGGAGGAG & CTGCCGTCAGATCACCAA & 369 \\
\hline 5 & TGTCTTGCAGTCGGAGAGAG & GGTGGACTTCTGTGTGTCGT & 493 \\
\hline 6 & AATTCGCCCAAACAACACA & CAGAGAAATCGCCTGGAAGT & 370 \\
\hline 7 & TGGCTGATGAACTTTTGCAC & TGGTTTAAATTTGGCAATTCAT & 392 \\
\hline 8.1 & AGCTGTAGGCTGCAATCTGG & GTGGCAATCAGGTTTCACGT & 550 \\
\hline 8.2 & TTTTGCAAAGCCAGCTGACT & TAGGCTGCGAAGACCAGAAA & 468 \\
\hline 8.3 & GAATTGTCCCAGCATGACCT & TAGAACAGTCTGCTTGCCCAA & 497 \\
\hline
\end{tabular}

Table II. Polymerase chain reaction primer sequence of the exons of microphthalmia-associated transcription factor gene.

\begin{tabular}{|c|c|c|c|}
\hline Exon & $\begin{array}{l}\text { Sequence of upstream } \\
\text { primer }\left(5^{\prime}-3^{\prime}\right)\end{array}$ & $\begin{array}{l}\text { Sequence of downstream } \\
\text { primer }\left(5^{\prime}-3^{\prime}\right)\end{array}$ & $\begin{array}{l}\text { Fragment } \\
\text { size (bp) }\end{array}$ \\
\hline 1 & TGGTGTCTCGGGATACCTTG & TGGCATCAAATAATAAACAGCA & 304 \\
\hline 2 & GGCCTGGTCAGTTTCATGTT & GGGGACAAAGGCTGGTAAAT & 449 \\
\hline 3 & CATCTTGTTGCTGTGCCATC & TGGCAATGTTCCTAGCTTTCT & 324 \\
\hline 4 & GACCATTATTGCTTTGGGTAAAA & TGTGATCCTGAGATAATTCTCCATT & 343 \\
\hline 5 & CAAAGGGAACTGGTTGAGGA & TGTTTTAACCACTGCAGAGACC & 464 \\
\hline 6 & GCTTTTGAAAACATGCAAGC & TTGGTTTCTCTCAAATACAATTTTC & 398 \\
\hline 7 & CATGACCTGGAGAAGTTAATATGC & AGTGTCCAACAATCCTTTTGC & 398 \\
\hline 8 & CACCTGTTCCCCCAAAACTA & GTCAACTCCCCTATGGCTCA & 372 \\
\hline 9 & CTAATGACGCGCATCTACCA & TCAAGAAAACCCCTTCAGGT & 594 \\
\hline
\end{tabular}

Table III. Polymerase chain reaction primer sequence of the exons of sex-determinging region Y-box 10 gene.

\begin{tabular}{clll}
\hline Exon & \multicolumn{1}{c}{$\begin{array}{c}\text { Sequence of upstream } \\
\text { primer }\left(5^{\prime}-3 '\right)\end{array}$} & $\begin{array}{c}\text { Sequence of downstream } \\
\text { primer (5'-3') }\end{array}$ & $\begin{array}{c}\text { Fragment } \\
\text { size }(\mathrm{bp})\end{array}$ \\
\hline 2 & GTGGGCGTTGGACTCTTTGC & GCCTCGGGCTACCCTGAATCC & 587 \\
3 & CCCAGGGCCTCACATCTTCC & CATTGCCATCCAGCCATCTCC & 453 \\
4.1 & ACCTGCCTCTAACCTGCTTCCT & ATAATAGGGTCCTCCTGAGGGCTGATG & 597 \\
4.2 & ACTACACCGACCAGCCATCC & GCAGTGAGCCAGACAGAAAGC & 452 \\
\hline
\end{tabular}

Table IV. Polymerase chain reaction primer sequence of the exons of snail family zinc finger 2 gene.

\begin{tabular}{llll}
\hline Exon & $\begin{array}{c}\text { Sequence of upstream } \\
\text { primer }\left(5^{\prime}-3^{\prime}\right)\end{array}$ & $\begin{array}{c}\text { Sequence of downstream } \\
\text { primer }\left(5^{\prime}-3^{\prime}\right)\end{array}$ & $\begin{array}{c}\text { Fragment } \\
\text { size }(\mathrm{bp})\end{array}$ \\
\hline 1 & GCTGTGATTGGATCTTTCTTGC & TGTAAGCTCCCTTTCAGGACAC & 449 \\
2 & TGTGTGTATACTTGCGTGTGG & CTTCATGCAAATCCAACAGCC & 700 \\
3 & ATTTCTGTATGATTGGCAGCAG & GCTTCGGAGTGAAGAGAAATC & 471 \\
\hline
\end{tabular}

buffer (TaKaRa Biotechnology Co. Ltd., Dalian, China) and specific primers. The reaction was then performed for
30 cycles under $95^{\circ} \mathrm{C}$ for $30 \mathrm{sec}, 60^{\circ} \mathrm{C}$ for $30 \mathrm{sec}$, and $72^{\circ} \mathrm{C}$ for $30 \mathrm{sec}$ on an ABI 9700 thermal cycler (Applied Biosystems; 
A

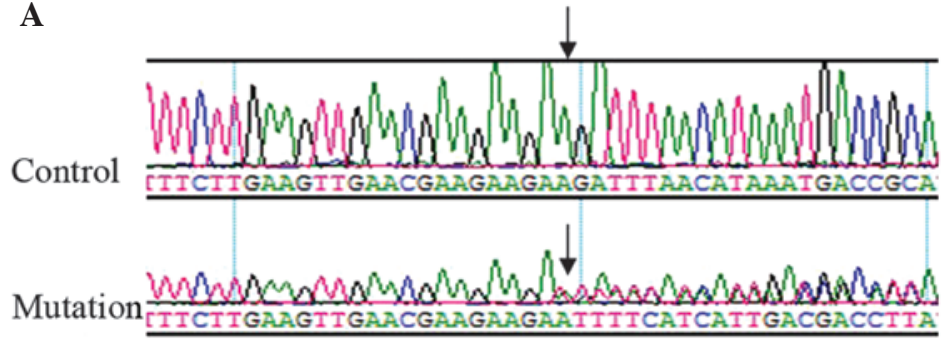

B

Control

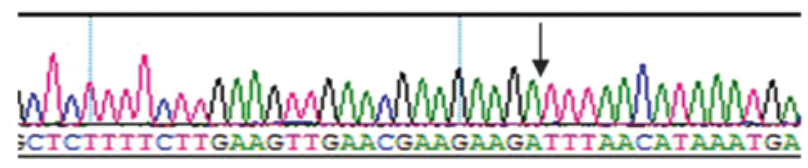

Mutation

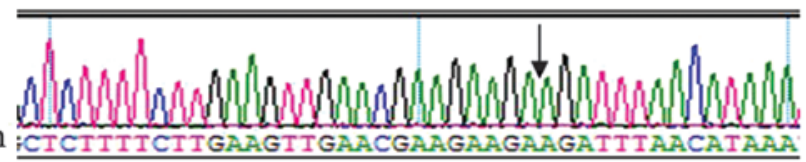

c.649_651delAGA

Figure 4. Mutation analysis of Chinese WS pedigree WS01. (A) Genomic DNA sequence demonstrating loss of heterozygosity mutations compared with the wild-type control. (B) DNA sequence following cloning of exon 7 of the MITF gene. The structure of MITF indicates the position of c.649_651delAGA mutation in exon 7. WS, Waardenburg syndrome; MITF, microphthalmia-associated trancription factor.

Thermo Fisher Scientific, Inc., Waltham, MA, USA). Following amplification, the PCR products were subjected to agarose gel electrophoresis and purified using MiniBest Agarose Gel DNA Extraction kit Ver4.0 (TaKaRa Biotechnology Co. Ltd.). The upstream and downstream amplifying primers were used for sequence detection by Sanger sequencing on a 3730 DNA analyzer (Applied Biosystems; Thermo Fisher Scientific, Inc.). The gene sequences were compared and analyzed using GenBank (http://www.ncbi.nlm.nih.gov/genbank/), in order to identify any mutations. Sanger sequencing was conducted for verification on all family members

\section{Results}

Pedigree analysis. Pedigree 1 consisted of three generations (nine persons) and five patients with WS. The following characteristics were observed: (i) The disease was observed in each subsequent generation following the proband's grandfather; (ii) one of the patient's parents was affected; (iii) the offspring of individuals without the disease did not suffer from the disease; (iv) and male and female children exhibited the same probability of suffering from the disease. Therefore, WS in pedigree 1 exhibited an autosomal dominant inheritance pattern. The mode of inheritance of WS in pedigrees 2 and 3 remains to be confirmed as there weren't enough patients in these families to gain reliable results.

Clinical diagnosis. The proband in pedigree 1 had blue irises, prelingual deafness and hearing loss without progressive aggravation. Acoustic conductance detected 'A' type wave, and ossa temporale CT and MRI demonstrated an increase in density in the middle ear cavity. Chronic otomastoiditis and traumatic brain injury were not observed on the MRI, with no other major abnormality. Both ears did not pass the distortion product otoacoustic emission (DPOAE) test, suggesting

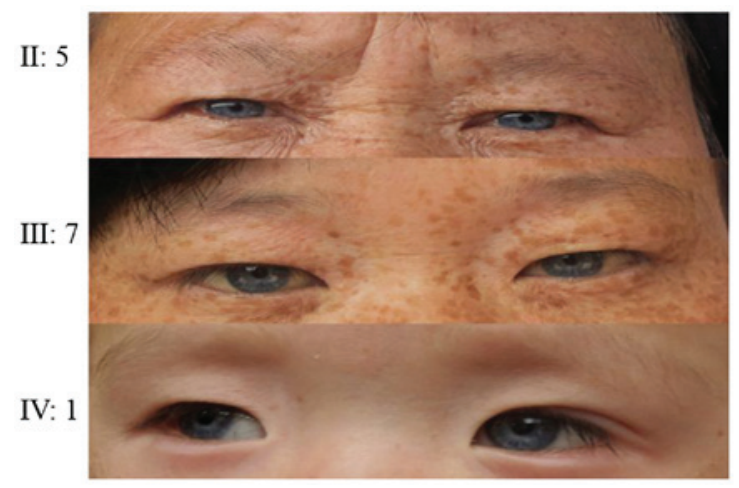

Figure 5. Iris color of Chinese WS pedigree WS01 cases. WS, Waardenburtg syndrome.

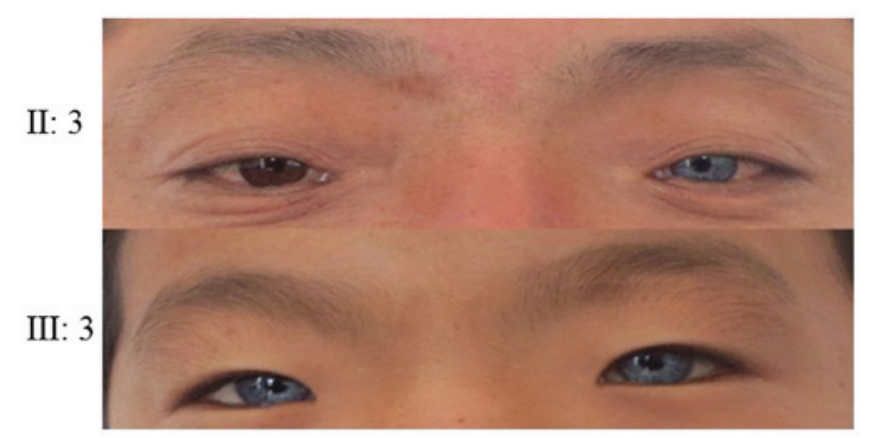

Figure 6. Iris color of Chinese WS pedigree WS02 cases. WS, Waardenburg syndrome.

pathology of the cochlea. ABR, multi-frequency stable evoked potential, and acoustic field measurement results suggested severe neurosensory deafness in the proband. Probands of pedigrees 2 and 3 had blue irises, prelingual deafness and 
hearing loss without progressive aggravation (Figs. 5-7). Acoustic conductance detected 'A' type wave and ossa temporale $\mathrm{CT}$ and high-resolution MRI observed no abnormality, suggesting a normal middle ear. Both ears did not pass the DPOAE test, suggesting pathology of the cochlea. ABR, multi-frequency stable evoked potential, and acoustic field measurement results suggested severe neurosensory deafness in both ears of the probands. According to the WS diagnostic standards (W index values should be $<1.95$ ) produced by Faarer et al (3) and Liu et al (4) in 1995, which is recommended by the Waardenburg association, the $\mathrm{W}$ index values of the probands in the present study were all $<1.95$, and probands also demonstrated congenital neurosensory deafness, different colored irises, and no angulus oculi medialis translocation, thus suggesting all three families conform to the WS type II diagnosis.

Analysis of mutations in candidate disease-causing genes. Genetic analysis of the proband of pedigree 1 observed mixed peaks in exon 7 of the MITF candidate gene; however, the unrelated relatives exhibited a single peak, suggesting a loss of heterozygosity (Fig. 4A). Sequence detection revealed the c.649_651delAGA mutation (Fig. 4B). Members II:5, III:7 and III:9 of pedigree 1 all exhibited the same genetic mutation. Following exon detection of the candidate mutant genes, PAX3, SOX10 and SNAI2 in family 1, no disease-causing mutations were observed in these genes. Following exon detection of the candidate genes, PAX3, MITF, SOX10 and SNAI2 in family 2 and family 3 , no disease-causing genetic mutations were observed.

\section{Discussion}

The five predominant diagnostic criteria for WS are: (i) Congenital sensorineural hearing loss; (ii) iris pigment abnormalities, including complete heterochromia iridis where the eyes are different colors, partial or fragmentary heterochromia iridis where blue is present in parts of the iris, and bright blue irises; (iii) hypochromic change of hair, presented by white hair on the forehead; (iv) no angulus oculi medialis malposition; (v) and a first-degree relative with the condition. WS type II is diagnosed by the presence of two of the five predominant diagnostic criteria with no angulus oculi medialis malposition. Following observation of the probands from the three pedigrees investigated in the present study, they all exhibited the three most common and marked phenotypic characteristics of WS type II: Sensorineural prelingual deafness, bright blue irises, white hair at the forehead, and $\mathrm{W}$ indexes $<1.95$, thus suggesting that all probands had WS type II.

Due to the genetic heterogeneity and reduced penetrance of WS, differences are often observed between patients from both different families and the same family (5). Among the nine patients in the three families tested in the present study, deafness and different iris colors were the most common clinical phenotypes of WS, with a penetrance of $77 \%(7 / 9$ cases) and 88\% (8/9 cases), respectively. Facial freckles and pigmentation on the neck, chest and abdomen, and upper limbs were observed in II:5, II:7 and III:9 in family 1. The father of the proband in family 2 had one blue iris, premature white

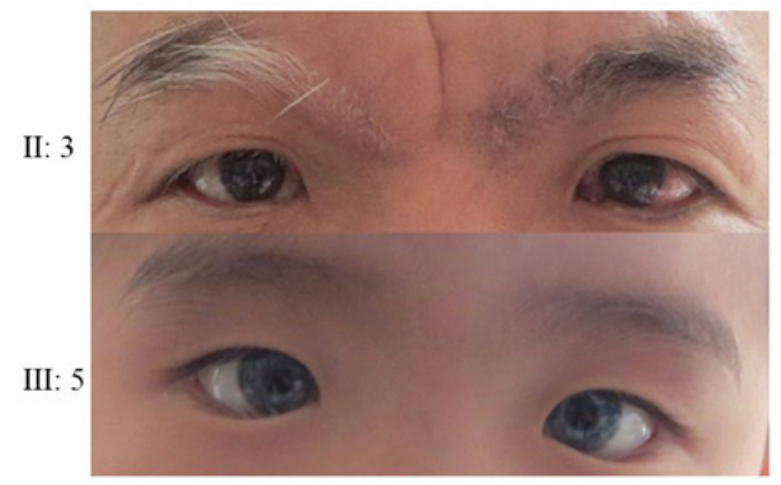

Figure 7. Iris color of Chinese WS pedigree WS03 cases. WS, Waardenburg syndrome.

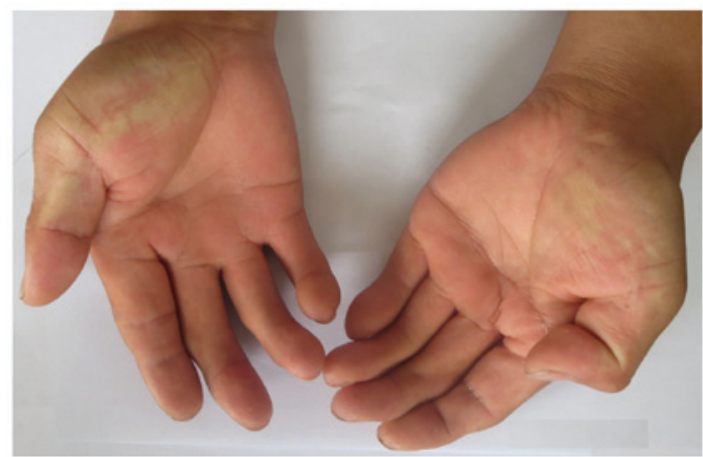

Figure 8. Finger joints of the proband's father (II:3) in Chinese WS pedigree WS02. WS, Waardenburg syndrome.

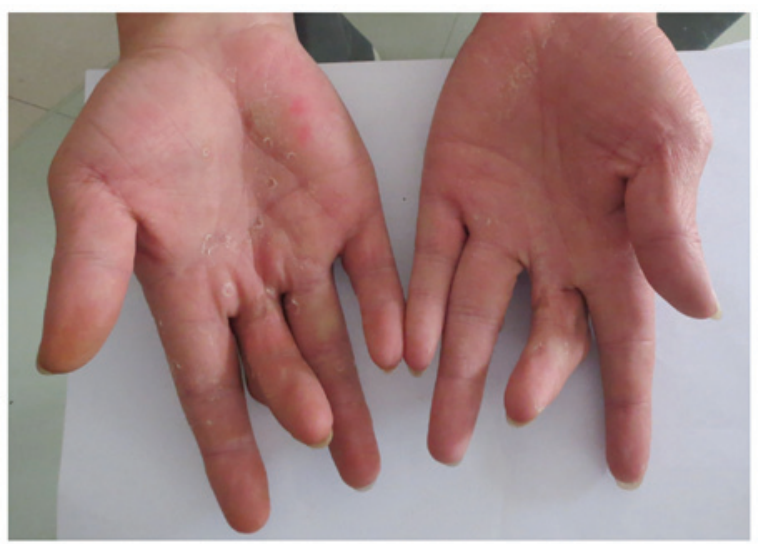

Figure 9. Finger joints of the proband's father (II:3) in Chinese WS pedigree WS03. WS, Waardenburg syndrome.

hair, mild deformity of the left elbow joint, limited extension and mild fusion abnormality at the extreme ends of his middle fingers, fourth fingers and little fingers (Fig. 8), but no clinical phenotype of deafness. The father of the proband in family 3 exhibited normal iris color and hearing, with no abnormal skin pigmentation but limited extension of middle fingers (Fig. 9). His hair, right eyebrow and right eyelashes were white by the age of 30 . The other members of the two families exhibited no abnormalities of limb joints, or the skeletal or digestive systems, thus excluding WS type III, despite the overlapping characteristics of each subtype. The known relevant genes in 
WS are positioned on chromosomes 2, 3, 8, 13, 20 and 22, and are PAX3, MITF, SNAI2, endothelin 3, endothelin receptor type B and SOX10, respectively (6). PAX3 is the predominant disease-causing gene of WS types I and III (7). The pathogenesis of WS type II is associated with genetic mutations of MITF, SNAI2 and SOX10 (8-10); therefore, PAX3, MITF, SOX10 and SNAI2 were considered the candidate disease-causing genes in the three families of the present study, and were the focus of mutation detection.

MITF is expressed in melanocytes and encodes a transcription factor containing the helix-loop-helix-leucine zipper structure. It is also a key factor in regulating the growth of melanocytes. Defects in melanocytes result in abnormal pigment distribution, whereas defects in melanocytes of the stria vascularis lead to deafness (11). Genetic mutation analysis demonstrated that all patients in pedigree 1 had c.649_651AGA fragment loss in exon 7 of the MITF candidate gene. This mutation results in the loss of codon 217 encoding arginine, and does not appear in normal controls. Arg217 mutation influences the positioning of MITF protein in the cell nucleus and inhibits wild-type MITF function (12), which is consistent with the cases in family $1(13,14)$.

MITF gene mutation is also observed in Tietz syndrome (albinism-deafness syndrome) (15). All patients in pedigree 1 exhibited congenital severe sensorineural hearing loss, bright blue irises, marked brown freckle deposition on the face, neck and/or limbs, but no whole-body pigmentation alterations; therefore, Tietz syndrome was excluded. Detection of known candidate disease-causing genes (MITF, SOX10, SNAI2 and PAX3) was also conducted on the patients in pedigrees 2 and 3; however, no known genetic mutations associated with WS were observed. At present, MITF and SOX10 mutations are detected in $30 \%$ of patients with WS type II $(8,10)$. Yang et al (16) reported that the genetic mutation rate of MITF in China may be higher than in non-Asian people. The majority of cases of WS type II cannot be diagnosed at a molecular level, thus suggesting that there are other disease-causing genetic mutations requiring further study that are important in the pathogenesis of WS.

Previous studies demonstrated that following treatment with the electronic cochlear implant, the rehabilitation efficacy in patients with WS is comparable to other deaf patients $(17,18)$. Following the relevant examination of the probands of pedigrees 1 and 3 , an electronic cochlear implant was fitted. Following the surgery, the patients reacted to sound and underwent language rehabilitation; however, the rehabilitation effects require further follow-up.

In conclusion, no mutations were identified in the relevant genes, including PAX3, MITF, SOX10 and SNAI2 in two of the three families with type II WS, which implies that novel pathogenic mutations associated with type II WS may exist and require further investigation.

\section{Acknowledgements}

The present study was supported by the Science and Technology project of Hunan Province (grant no. 2014SK3020) and Major National Scientific Research Projects (grant no. 2012CB967904).

\section{References}

1. Kochhar A, Hildebrand MS and Smith RJ: Clinical aspects of hereditary hearing loss. Genet Med 9: 393-408, 2007.

2. Read AP and Newton VE: Waardenburg syndrome. J Med Genet 34: 656-665, 1997.

3. Farrer LA, Grundfast KM, Amos J, Arnos KS, Asher JH Jr, Beighton P, Diehl SR, Fex J, Foy C, Friedman TB, et al: Waardenburg syndrome (WS) type I is caused by defects at multiple loci, one of which is near ALPP on chromosome 2: First report of the WS consortium. Am J Hum Genet 50: 902-913, 1992.

4. Liu XZ, Newton VE and Read AP: Waardenburg syndrome type II: Phenotypic findings and diagnostic criteria. Am J Med Genet 55: 95-100, 1995.

5. Farrer LA, Arnos KS, Asher JH Jr, Baldwin CT, Diehl SR, Friedman TB, Greenberg J, Grundfast KM, Hoth C, Lalwani AK, et al: Locus heterogeneity for Waardenburg syndrome is predictive of clinical subtypes. Am J Hum Genet 55: 728-737, 1994.

6. Pingault V, Ente D, Dastot-Le Moal F, Goossens M, Marlin S and Bondurand N: Review and update of mutations causing Waardenburg syndrome. Hum Mutat 31: 391-406, 2010.

7. Hoth CF, Milunsky A, Lipsky N, Sheffer R, Clarren SK and Baldwin CT: Mutations in the paired domain of the human PAX3 gene cause Klein-Waardenburg syndrome (WS-III) as well as Waardenburg syndrome type I (WS-I). Am J Hum Genet 52: 455-462, 1993.

8. Bondurand N, Dastot-Le MF, Stanchina L, Collot N, Baral V, Marlin S, Attie-Bitach T, Giurgea I, Skopinski L, Reardon W, et al: Deletions at the SOX10 gene locus cause Waardenburg syndrome types 2 and 4. Am J Hum Genet 81: 1169-1185, 2007.

9. Sánchez-Martín M, Rodríguez-García A, Pérez-Losada J, Sagrera A, Read AP and Sánchez-García I: SLUG (SNAI2) deletions in patients with Waardenburg disease. Hum Mol Genet 11: 3231-3236, 2002.

10. Tassabehji M, Newton VE and Read AP: Waardenburg syndrome type 2 caused by mutations in the human microphthalmia (MITF) gene. Nat Genet 8: 251-255, 1994.

11. Curran K, Raible DW and Lister JA: Foxd3 controls melanophore specification in the zebrafish neural crest by regulation of Mitf. Dev Biol 332: 408-417, 2009.

12. Takebayashi K, Chida K, Tsukamoto I, Morii E, Munakata H, Arnheiter H, Kuroki T, Kitamura Y and Nomura S: The recessive phenotype displayed by a dominant negative microphthalmia-associated transcription factor mutant is a result of impaired nucleation potential. Mol Cell Biol 16: 1203-1211, 1996.

13. Chen H, Jiang L, Xie Z, Mei L, He C, Hu Z, Xia K and Feng Y: Novel mutations of PAX3, MITF, and SOX10 genes in Chinese patients with type I or type II Waardenburg syndrome. Biochem Biophys Res Commun 397: 70-74, 2010.

14. Tassabehji M, Newton VE, Liu XZ, Brady A, Donnai D, Krajewska-Walasek M, Murday V, Norman A, Obersztyn E, Reardon W, et al: The mutational spectrum in Waardenburg syndrome. Hum Mol Genet 4: 2131-2137, 1995.

15. Smith SD, Kelley PM, Kenyon JB and Hoover D: Tietz syndrome (hypopigmentation/deafness) caused by mutation of MITF. J Med Genet 37: 446-448, 2000.

16. Yang S, Dai P, Liu X, Kang D, Zhang X, Yang W, Zhou C, Yang S and Yuan H: Genetic and phenotypic heterogeneity in Chinese patients with Waardenburg syndrome type II. PLoS One 8: e77149, 2013.

17. Amirsalari S, Ajallouyean M, Saburi A, Haddadi Fard A, Abed M and Ghazavi Y: Cochlear implantation outcomes in children with Waardenburg syndrome. Eur Arch Otorhinolaryngol 269: 2179-2183, 2012.

18. Kontorinis G, Lenarz T, Giourgas A, Durisin M and Lesinski-Schiedat A: Outcomes and special considerations of cochlear implantation in waardenburg syndrome. Otol Neurotol 32: 951-955, 2011. 